\title{
Aerobic Exercise Effect on Improving Metabolism after Burn Injury
}

\author{
FATEMA M. ABDALLA, M.Sc.; SAMAH H. NAGIB, Ph.D. and NESSRIEN A. ABDELRASHID, Ph.D. \\ The Department of Surgery, Faculty of Physical Therapy, Cairo University
}

\begin{abstract}
Background: Disturbance of metabolism is a common sign that is largely detected after trauma or burn injury and characterized by impaired organ functioning and wound healing. The disparity in research and the necessity to add to the existing literature base led to the development of this study.

Aim of Study: To investigate if aerobic exercise can improve the metabolism after burn injury $10-15 \%$ TBSA in the body.

Material and Methods: Thirty burn patients with 10-15\% TBSA their age ranged from 20-40 years were selected from the inpatients Burn Department of Ismailia General Hospital, or any hospital in Ismailia. The thirty patients were classified into two groups equal in number; each group consisted of fifteen patients. The first received a program of aerobic exercise in addition to circulatory and respiratory exercises (Group A) 3 times/week for four weeks and the second received a program of circulatory and respiratory exercises (Group B) 3 times/week for four weeks. The changes in the metabolic markers were measured at the beginning of the study and after four weeks from 15 April 2017 to 15 May 2017.
\end{abstract}

Conclusion: It was concluded that the aerobic exercise is associated with significant improvement (increase) in metabolic markers greater than respiratory and circulatory exercise in burn patients in a short term (up to four weeks).

Key Words: Aerobic - Burn - Exercise - Metabolism - Total protein-Albumin-Circulatory-Respiratory.

\section{Introduction}

BURN is a type of injury to flesh or skin caused by heat, electricity, chemicals, friction, or radiation. Burns that affect only the superficial skin are known as superficial or first-degree burns. When damage penetrates into some of the underlying layers, it is a partial-thickness or second-degree burn. In a full-thickness or third-degree burn, the injury extends to all layers of the skin. A fourthdegree burn additionally involves injury to deeper tissues, such as muscle or bone [1]

Correspondence to: Dr. Fatema M. Abdalla, The Department of Surgery, Faculty of Physical Therapy, Cairo University
Anemia secondary to full thickness burns of greater than $10 \%$ TBSA is common. Electrical burns may lead to compartment syndrome or rhabdo-myolysis due to muscle breakdown. Blood clotting in the veins of the legs is estimated to occur in 6 to $25 \%$ of people. The hyper metabolic state that may persist for years after a major burn can result in a decrease in bone density and a loss of muscle mass. Keloids may form subsequent to a burn, particularly in those who are young and dark skinned. Following a burn, children may have significant psychological trauma and experience post-traumatic stress disorder. Scarring may also result in a disturbance in body image. In the developing world, significant burns may result in social isolation, extreme poverty and child abandonment [2]

The hyper metabolic and catabolic response after burn injury is similar to that seen following any major trauma; however: The severity of the response is greater than for any other form of trauma or during sepsis [3]

Aerobic exercise (also known as cardio) is physical exercise of low to high intensity that depends primarily on the aerobic energy-generating process. Aerobic literally means "relating to, involving, or requiring free oxygen", and refers to the use of oxygen to adequately meet energy demands during exercise via aerobic metabolism. Generally, light-to-moderate intensity activities that are sufficiently supported by aerobic metabolism can be performed for extended periods of time [4].

\section{Subjects and Methods}

This study was conducted at Ismailia General Hospital, or any Hospital in Ismailia, from 15 April to 15 May 2017. The study had been approved by Ethics Committee of the hospital. Thirty male and 
female patients was participated in this study; they were divided into two groups. Participants were excluded if they had been treated using chemo or radiotherapy. Patient who have associated disorders, pregnancy immunodeficiency, HIV, diabetes, anemia, chest disease, smokers or hepatic disease, cardiac disease.

The purpose and the procedures of the study were sufficiently explained to the participants, and their informed consent form was taken before the beginning of the study.

\section{I- Evaluative procedure:}

1- All patients came at $1 \mathrm{Pm}$ without eating at least 2 hours before session.

2- An informed consent was taken from all patients that will participate in the study (Appendix A).

3- Before starting the study all patients were informed about the nature, benefits and procedure of the study.

4- Plasma or serum samples were obtained by venipuncture (arterial cannula.

5- A venous blood sample was drawn from the anti-cubital vein of burned patient for 6 to 8 hours to detect serum level of total protein and albumin.

\section{II- Patients preparation:}

1-All patients were informed about the procedure before starting the session.

2- All patients of Group (A) were instructed not to eat at least two hours before walking exercise session to prevent injury and to prevent falling during walking.

3-All patients were instructed about the precautions to prevent injury:

- Clothing: Dressing comfortably and practically (for example, items that did not restrict movement and allow sweating easily).

- Personal feeling: No training if patient overtired or feeling ill.

- Breathing: Breathing normally while lifting by exhaling during the exertion or harder phase and inhaling during the easier or relaxation phase.

\section{III- Training procedures:}

\section{A- Material preparation:}

Calibration of the Treadmill according to the exercise program.

\section{B- Training:}

1- The steps of training were explained for each patient.

2- Patients in Group (A):

- Each patient practiced treadmill exercise training program at zero inclination with a moderate intensity for fifty minutes by increasing gradually by the end of the program three times per week for 1 month (4 weeks).

- This program included the following phases which are:

A- Warming up phase: Each patient practiced treadmill exercise for 5 minutes with lowest speed to enhance cardiovascular adjustment and prevent musculoskeletal injuries.

B- Stimulus phase: Each patient continued the treadmill exercise training with a moderate intensity from $40 \%$ to $60 \%$ of the pre-determined individualized maximum heart rate (HR$\max$ ) for thirty minutes.

C- Cool down phase: Each patient continued the treadmill exercise training for 5 minutes with lowest speed.

- Moderate intensity aerobic exercise parameters:

-Intensity: Training heart rate $=60 \%>\mathrm{MHR}>40 \%$. [MHR=220-age].

- Duration: (5min. for warming up, 30min. for training \& 5min. for cooling down).

- Frequency: 3 sessions/week.

- Mode of training: Walking program.

- All patients left the clinic after ending the session nearly at $2 \mathrm{Pm}$.

Steps of the motorized treadmill treatment procedures:

15 patients with burn injury $10-15 \%$ TBSA in the acute stage started walking program on the motorized treadmill for 4 weeks, with frequency of 3 times per week. Program was started by peace walking for $20 \mathrm{~min}$ at the first week then $30 \mathrm{~min}$ at the second week then 40min at the third week then $50 \mathrm{~min}$ at the fourth week.

N.B: The aerobic exercise was $40-60 \%$ of the max heart rate.

- This was beside the traditional physiotherapy program (circulatory and breathing exercises). 


\section{3- $\operatorname{Group}(B)$ :}

15 patients others with same injury and same stage started traditional physiotherapy program (circulatory and breathing exercises) only.

\section{Measurement procedures:}

1- Spectrophotometer.

2- Motorized treadmill.

\section{Results}

The main purpose of this study was to investigate the effect of aerobic exercises on improving the metabolism after burn injury.

The results of this study revealed that there was a highly statistical significant increase in albumin and total protein after aerobic exercises when compared with its corresponding value in control group.

Table (1): Comparative analysis of Albumin level between two groups of the study.

\begin{tabular}{|c|c|c|c|c|}
\hline & \multicolumn{4}{|c|}{ Albumin level } \\
\hline & \multicolumn{2}{|c|}{ Pre-treatment } & \multicolumn{2}{|c|}{ Post-treatment } \\
\hline & \multicolumn{4}{|c|}{ Group A Group B Group A Group B } \\
\hline $\bar{x}$ & 2.77 & 2.56 & 4.81 & 3.2 \\
\hline$\pm \mathrm{SD}$ & \pm 0.46 & \pm 0.49 & \pm 0.85 & \pm 0.50 \\
\hline MD & \multicolumn{2}{|c|}{0.21} & \multicolumn{2}{|c|}{1.61} \\
\hline$t$-value & \multicolumn{2}{|c|}{1.223} & \multicolumn{2}{|c|}{6.271} \\
\hline$p$-value & \multicolumn{2}{|c|}{0.231} & \multicolumn{2}{|c|}{0.0001} \\
\hline Level of significance & \multicolumn{2}{|c|}{ N.S } & \multicolumn{2}{|c|}{$\mathrm{S} \uparrow$} \\
\hline \multicolumn{5}{|l|}{$N . B$ : } \\
\hline $\begin{array}{l}\bar{x}: \text { Mean. } \\
\pm \mathrm{SD}: \text { Standard Deviat } \\
\mathrm{MD}: \text { Mean Difference }\end{array}$ & \multicolumn{4}{|c|}{$\begin{array}{ll}p \text {-value } & \text { Probability level. } \\
\text { N.S } & : \text { Non-Significance. } \\
\mathrm{S} & : \text { Significant. }\end{array}$} \\
\hline
\end{tabular}

Table (2): Comparative analysis of total protein between two groups of the study.

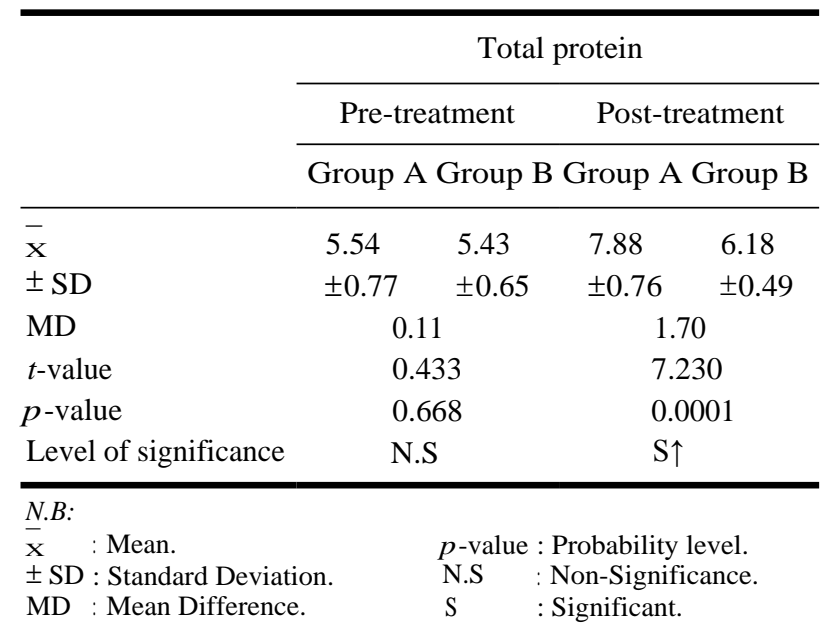

\section{Discussion}

The results of this study confirmed that anabolism (albumin and total protein) respond well to aerobic exercises after burn injury.

\section{In agreement with our results;}

Yang et al., [5], measured hepatic albumin synthesis in five volunteers ( 4 men and 1 woman) at 3 and $6 \mathrm{~h}$ after recovery from intense exercise. They measured an increase in hepatic albumin synthetic rate 3-6h after a single exposure of intense intermittent exercise. This elevation in albumin synthetic rate could produce the increased albumin expected at $24 \mathrm{~h}$ of recovery from exercise if the albumin synthetic rate increased progressively by $4 \% / \mathrm{h}$ over the 24 -h recovery period.

Thirty-five children, ages $7-17 \mathrm{yr}$ old, were enrolled in the study by Suman et al., [6], only patients with $>40 \%$ of Total Body Surface Area (TBSA) burned during excisional surgery in the acute phase of injury, were enrolled. Patients were randomized into two groups. One group was to participate in a 12-wk in-hospital physical rehabilitation program supplemented with an individualized and supervised exercise training program $(R E x ; n=19)$. The nonexercising group $(R ; n=16)$ was to participate in a 12-wk, home-based physical rehabilitation program without individualization and supervision of exercise. There is an increase in muscle strength, total work, and average power in the REx group after $12 \mathrm{wk}$ of exercise and that this increase is not observed in the R Group. In addition, there is a $>20$-fold difference in the mean rate of increase in total LBM in the REx Vs. R group, which paralleled the increase in muscle strength, total work, and power. But in this study no measurement of albumin or protein were done.

The study by De Lateur et al., [7], on 35 adult patients admitted to a burn center for treatment of a serious burn injury. They found that the aerobic capacity of adult burn survivors can be improved with participation in a structured, 12-week exercise program after injury.

Also, El-Hamid \& Ismail [8], their study was done on 70 subjects who were divided into two groups, Group 1 included 50 patients with type 2 diabetes mellitus and Group 2 consisted of 20 normal subjects. Both underwent aerobic exercise. They resulted that total serum protein and albumin were found to have significantly higher mean values post exercise than pre exercise in both groups of patients. 


\section{In contrast to our results;}

Afshar et al., [9], their studied groups were, resistance training $(n=7)$, moderate intensity aerobic training $(n=7)$, and control groups $(n=7)$. All undergoing conventional maintenance HD. Albumin (g/dL) levels were $4 \pm 0.3,4 \pm 0$ and $4 \pm 0.3$ at base line in control, aerobic exercise and resistance training groups respectively. While $4 \pm 0.3,4 \pm 0.4$ and $4 \pm 0.3$ at the end of study line in control, aerobic exercise and resistance training groups respectively. Exercises had no influence on serum albumin.

Also, Liu et al., [10], studied 22 patient received 12-week aerobic exercise program(control \& heomodialysis groups). The mean albumin level 3.45 $\pm 0.50 \mathrm{~g} / \mathrm{dl}$ for the control group and $3.45 \pm 0.28 \mathrm{~g} / \mathrm{dl}$ for the exercise group before the start of the exercise program; and $3.46 \pm 0.48 \mathrm{~g} / \mathrm{dl}$ for the control group and $3.52 \pm 0.28 \mathrm{~g} / \mathrm{dl}$ for the exercise group after the program ended. There was no significantly improving serum albumin level. Patients included in the study were CKD patients.

\section{Conclusion:}

The burned patients after aerobic exercise program combined with traditional physiotherapy showed a significant improvement in total protein and albumin than traditional physiotherapy only at the end of program.

So, that study showed clear benefits of the aerobic exercise in improving metabolic markers (total protein and albumin) greater than traditional physiotherapy in burned patients in a short term (up to four weeks).

\section{References}

1- HERNDON D. (ed.): "Chapter 3: Epidemiological, Demographic, and Outcome Characteristics of Burn Injury".
Total burn care (4th ed.). Edinburgh: Saunders, p. 23. ISBN 978-1-4377-2786-9, 2000.

2- ROJAS Y., FINNERTY C.C., RADHAKRISHNAN R.S and HERNDON D.N., (Decembre): "PinPharmacother 13 (17): 248594. Doi:10.1517 14656566. 2012. 738195.PMC 3576016. PMID 23121414, 2010.

3- JUCKETT G., HARTMAN-ADAMS H., (Aug. 1): "Management of keloids and hypertrophic scars". American family physician, 80 (3): 253-60. PMID 19621835, 2009.

4- GRANGER and JOYCE, (Jan.): "An Evidence-Based Approach to Pediatric Burns". Pediatric Emergency Medicine Practice, 6 (1), 2009.

5- YANG, ROGER C., GARY W. MACK, ROBERT R. WOLFE and ETHAN R. NADEL: Albumin synthesis after intense intermittent exercise in human subjects. J. Appl. Physiol., 84 (2): 584-92, 1998.

6- SUMAN O.E., SPIES R.J., CELIS M.M., MLCAK R.P. \& HERNDON D.N.: Effects of a 12-wk resistance exercise program on skeletal muscle strength in children with burn injuries. Journal of Applied Physiology, 91 (3): 1168-75, 2001.

7- De LATEUR B.J., MAGYAR-RUSSELL G., BRESNICK M.G., BERNIER F.A., OBER M.S., KRABAK B.J. and FAUERBACH J.A.: Augmented exercise in the treatment of deconditioning from major burn injury. Archives of physical medicine and rehabilitation, 88 (12): S18-S23, 2007.

8- EL-HAMID Y.M.A. and ISMAIL A.F.: Response of Liver Function Tests to Aerobic Exercise in Patients with Type 2 Diabetes Mellitus. The Medical Journal of Cairo University, 78 (2), 2010.

9- AFSHAR R., SHEGARFY L., SHAVANDI N. and SANAVI S.: Effects of aerobic exercise and resistance training on lipid profiles and inflammation status in patients on maintenance hemodialysis. Indian Journal of Nephrology, 20 (4): 185, 2010.

10- LIU Y.M., CHUNG Y.C., CHANG J.S. and YEH M.L. Effects of aerobic exercise during hemodialysis on physical functional performance and depression. Biological Research for Nursing, 17 (2): 214-21, 2015.

\section{فاعلية ممارسة التمرينات الهوائية

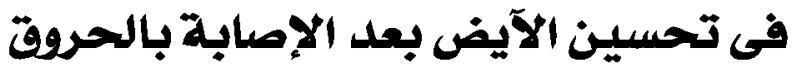

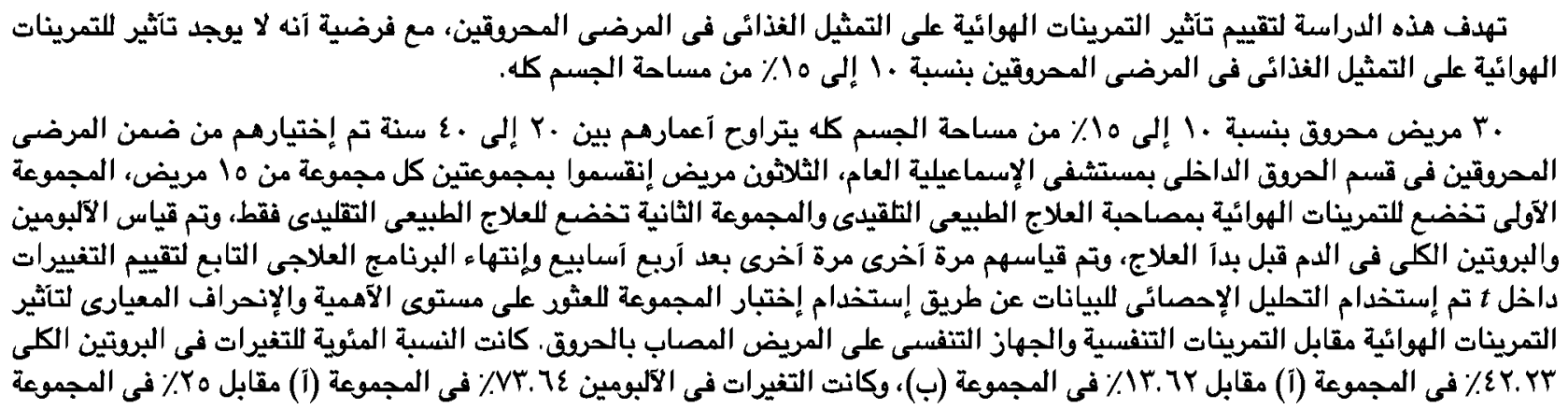

